\title{
Erratum to: Design and conduct of the activated protein $C$ and corticosteroids for human septic shock (APROCCHSS) trial
}

\author{
Djillali Annane ${ }^{1 *}$, Christian Brun-Buisson ${ }^{2}$, Alain Cariou ${ }^{3}$, Claude Martin $^{4}$, Benoit Misset $^{5}$, Alain Renault ${ }^{6}$, \\ Blandine Lehmann ${ }^{7}$, Valérie Millul ${ }^{8}$, Virginie Maxime ${ }^{1}$, Eric Bellissant ${ }^{6}$ and The APROCCHSS Investigators \\ for the TRIGGERSEP Network
}

\section{Erratum to: Ann. Intensive Care (2016) 6:43 DOI 10.1186/s13613-016-0147-3}

The original version of this article [1] was published with an incomplete version of the table listing "Study Centers and Investigators" found in the Appendix: Study organization.

The full and correct version of this table has been provided below.

\section{Study centers and investigators}

\begin{tabular}{ll}
\hline $\begin{array}{l}\text { Surname and name of } \\
\text { sites principal investigator }\end{array}$ & Study sites details \\
\hline ANNANE, Djillali & Service de Réanimation médicale \\
FADEL Fouad & HOPITAL RAYMOND POINCARE \\
POLITO Andrea & 104 BD RAYMOND POINCARE \\
CLAIR Bernard & 92380 GARCHES \\
MAXIME Virginie & \\
LUIS David & \\
QUENOT Jean-Pierre & Service de réanimation \\
& 14 RUE GAFFAREL BP 77908 21079 DIJON \\
& CEDEX \\
& HÔPITAL F. MITTERRAND \\
& CHU DE DIJON \\
& Service de Réanimation Médicale et \\
MEGARBANE, Bruno & Toxicologique \\
& Hôpital LARIBOISIERE \\
& 2 rue AMBROISE PARE \\
& 75010 PARIS \\
& Service d'Anesthésie-réanimation du Dr. \\
& Lorenzo \\
CENTRE HOSPITALIER D'ETAMPES \\
PERCHERON Stéphanie & 26 AV CHARLES DE GAULLE \\
& 91150 ETAMPES \\
\hline
\end{tabular}

*Correspondence: djillali.annane@aphp.fr

${ }^{1}$ General ICU, Service de Réanimation, Hôpital Raymond Poincaré, Laboratory of Infection and Inflammation, U1173, AP-HP, University of Versailles SQY and INSERM, 104 Boulevard Raymond Poincaré, 92380 Garches, France

Full list of author information is available at the end of the article

\begin{tabular}{|c|c|}
\hline $\begin{array}{l}\text { Surname and name of } \\
\text { sites principal investigator }\end{array}$ & Study sites details \\
\hline $\begin{array}{l}\text { CARIOU Alain } \\
\text { NGUYEN Yen-Lan } \\
\text { DAVIAUD Fabrice } \\
\text { BOUGLE Adrien } \\
\text { MIRA Jean Paul } \\
\text { CHICHE Jean Daniel } \\
\text { PENE Frederic } \\
\text { MORICHAU-BEAUCHANT } \\
\text { Tristan } \\
\text { GERI Guillaume }\end{array}$ & $\begin{array}{l}\text { Service de réanimation médicale } \\
\text { HOPITAL COCHIN } \\
\text { 27 R FAUBOURG ST-JACQUES } \\
75014 \text { PARIS }\end{array}$ \\
\hline $\begin{array}{l}\text { SCHWEBEL Carole } \\
\text { ARA SOMOHANO Claire } \\
\text { MINET Clémence } \\
\text { LUGOSI Maxime } \\
\text { CARTIER Jean Charles } \\
\text { POTTON Leïla }\end{array}$ & $\begin{array}{l}\text { Service de Réanimation médicale } \\
\text { CHU DE GRENOBLE } \\
\text { Avenue du Maquis du Gresivaudan, Pavil- } \\
\text { lon Dauphiné } \\
\text { BP } 217 \\
38043 \text { GRENOBLE }\end{array}$ \\
\hline $\begin{array}{l}\text { FORCEVILLE, Xavier } \\
\text { KUBA-KUSUTI Jean-Kamiena } \\
\text { TOUATI Samia }\end{array}$ & $\begin{array}{l}\text { Service de Réanimation Polyvalente } \\
\text { CENTRE HOSPITALIER DE MEAUX } \\
6 \text { R SAINT FIACRE } \\
77100 \text { MEAUX }\end{array}$ \\
\hline $\begin{array}{l}\text { REIGNIER Jean } \\
\text { COLIN Gwenhaël } \\
\text { MARTIN-LEFEVRE Laurent } \\
\text { BACHOUMAS Konstantinos } \\
\text { HENRY-LAGARRIGUE Matthieu } \\
\text { YEHIA Aihem } \\
\text { LASCARROU Jean-Baptiste } \\
\text { LEBERT Christine } \\
\text { LACHERADE Jean-Claude }\end{array}$ & $\begin{array}{l}\text { Service de réanimation } \\
\text { CENTRE HOSPITALIER DEPARTEMENTAL } \\
\text { SITE DE LA ROCHE-SUR-YON } \\
\text { LES OUDAIRIES } \\
85925 \text { LA ROCHE SUR YON CEDEX } 9\end{array}$ \\
\hline $\begin{array}{l}\text { ASEHNOUNE Karim } \\
\text { LOUTREL Olivier } \\
\text { DUMONT Romain } \\
\text { ROQUILLY Antoine } \\
\text { MAHE Pierre-Joachim } \\
\text { DEMEURE DIT LATTE } \\
\text { Dominique } \\
\text { CHAMPIN Philippe } \\
\text { ARNOULD Jean François } \\
\text { CINOTTI Raphaël } \\
\text { LE FLOCH Ronan }\end{array}$ & $\begin{array}{l}\text { Service d'Anesthésie Réanimation } \\
\text { chirurgicale, } \\
\text { CHU DE NANTES } \\
19 \text { RUE GENERAL MARGUERITTE, } 44000 \\
\text { NANTES }\end{array}$ \\
\hline
\end{tabular}

\section{Springer Open}

(0) 2016 The Author(s). This article is distributed under the terms of the Creative Commons Attribution 4.0 International License (http://creativecommons.org/licenses/by/4.0/), which permits unrestricted use, distribution, and reproduction in any medium, provided you give appropriate credit to the original author(s) and the source, provide a link to the Creative Commons license, and indicate if changes were made. 


\begin{tabular}{|c|c|}
\hline $\begin{array}{l}\text { Surname and name of } \\
\text { sites principal investigator }\end{array}$ & Study sites details \\
\hline $\begin{array}{l}\text { MERCIER Emmanuelle } \\
\text { GAROT Denis } \\
\text { DEQUIN Pierre François } \\
\text { PERROTIN Dominique } \\
\text { LEGRAS Annick } \\
\text { MANKIKIAN Julie } \\
\text { TALEC Patrice } \\
\text { EHRMANN Stephan } \\
\text { JORET Aurélie } \\
\text { LHOMMET Claire } \\
\text { JORET Aurélie } \\
\text { LHOMMET Claire } \\
\text { ROUVE Emmanuelle } \\
\text { BODET-CONTENTIN Laetitia } \\
\text { JOUAN Youenn } \\
\text { SALMON-GANDONNIERE } \\
\text { Charlotte }\end{array}$ & $\begin{array}{l}\text { Service de réanimation polyvalente, } \\
\text { CHU BRETONNEAU, } \\
\text { 2, BOULEVARD TONNELLE, } \\
\text { 37044 TOURS }\end{array}$ \\
\hline $\begin{array}{l}\text { ANTONINI, François } \\
\text { MARTIN Claude Denis } \\
\text { RAGONNET Benoît }\end{array}$ & $\begin{array}{l}\text { Service d'Anesthésie Réanimation } \\
\text { HOPITAL NORD } \\
\text { Chemin des BOURRELY } \\
13015 \text { MARSEILLE }\end{array}$ \\
\hline $\begin{array}{l}\text { BRUN BUISSON, Christian } \\
\text { RAZAZI Keyvan } \\
\text { DE PROST Nicolas } \\
\text { CARTEAUX Guillaume }\end{array}$ & $\begin{array}{l}\text { Service de Réanimation Médicale } \\
\text { Hôpital HENRI MONDOR } \\
51 \text { AV DE LATTRE DE TASSIGNY } \\
94010 \text { CRETEIL }\end{array}$ \\
\hline $\begin{array}{l}\text { TIMSIT, Jean François } \\
\text { MOURVILLIER Bruno }\end{array}$ & $\begin{array}{l}\text { Service de Réanimation - Maladies } \\
\text { Infectieuses } \\
\text { Hôpital BICHAT CLAUDE BERNARD } \\
46 \text { Rue HENRI HUCHARD } \\
75018 \text { PARIS }\end{array}$ \\
\hline $\begin{array}{l}\text { CHAGNON, Jean-Luc } \\
\text { ALI BEN ALI Mohamed }\end{array}$ & $\begin{array}{l}\text { Service de Réanimation Polyvalente } \\
\text { C.H. DE VALENCIENNES } \\
114 \text { AV DESANDROUINS } \\
\text { BP } 479 \\
59322 \text { VALENCIENNES }\end{array}$ \\
\hline $\begin{array}{l}\text { CHIMOT Loic } \\
\text { DELOUR Pierre } \\
\text { DESSALLES Pierre Henri } \\
\text { MONSEAU Yannick } \\
\text { SAINT-LEGER Mélanie } \\
\text { BEDON-CARTE Sandrine }\end{array}$ & $\begin{array}{l}\text { Service de Réanimation } \\
\text { CENTRE HOSPITALIER PERIGUEUX } \\
\text { 80, AVENUE GEORGES POMPIDOU } \\
\text { CS } 61205 \\
24019 \text { PERIGUEUX CEDEX }\end{array}$ \\
\hline $\begin{array}{l}\text { BOULAIN Thierry } \\
\text { MATHONNET Armelle } \\
\text { BRETAGNOL Anne } \\
\text { RUNGE Isabelle } \\
\text { BARBIER François } \\
\text { MULLER Gregoire }\end{array}$ & $\begin{array}{l}\text { Service de réanimation polyvalente } \\
\text { CHR D'ORLEANS } \\
\text { 1, RUE PORTE-MADELEINE - } \\
\text { 45000ORLEANS }\end{array}$ \\
\hline $\begin{array}{l}\text { FRANCOIS Bruno } \\
\text { CLAVEL Marc } \\
\text { VIGNON Philippe } \\
\text { PICHON Nicolas } \\
\text { FEDOU Anne-Laure } \\
\text { CHAPELLAS Catherine } \\
\text { GALY Antoine }\end{array}$ & $\begin{array}{l}\text { Service de réanimation polyvalente } \\
\text { CHU LIMOGES } \\
\text { 2, AVENUE MARTIN LUTHER KING } 87042 \\
\text { LIMOGES }\end{array}$ \\
\hline $\begin{array}{l}\text { MISSET, Benoit } \\
\text { GARROUSTE ORGEAS Maité } \\
\text { PHILIPPART François }\end{array}$ & $\begin{array}{l}\text { Service de Réanimation médicale } \\
\text { polyvalente } \\
\text { HOPITAL SAINT-JOSEPH } \\
\text { 185 R RAYMOND LOSSERAND } \\
75674 \text { PARIS }\end{array}$ \\
\hline $\begin{array}{l}\text { SOUWEINE, Bertrand } \\
\text { AIT-HSSAIN Ali }\end{array}$ & $\begin{array}{l}\text { Service de Réanimation } \\
\text { CHU CLERMONT-FERRAND } \\
58 \text { RUE MONTALEMBERT } \\
\text { BP69 } \\
63003 \text { CLERMONT FERRAND }\end{array}$ \\
\hline
\end{tabular}

\begin{tabular}{|c|c|}
\hline $\begin{array}{l}\text { Surname and name of } \\
\text { sites principal investigator }\end{array}$ & Study sites details \\
\hline CHARPENTIER, Claire & $\begin{array}{l}\text { Service de Réanimation chirurgicale } \\
\text { HOPITAL CENTRAL } \\
29 \text { AVENUE DU MARECHAL DE } \\
\text { LATTRE DE TASSIGNY } \\
54037 \text { NANCY }\end{array}$ \\
\hline $\begin{array}{l}\text { MIGNON, Alexandre } \\
\text { BAUDIN Francois } \\
\text { ANTONA Marion } \\
\text { MEGHENEM Alia } \\
\text { DEMESMAY Marine }\end{array}$ & $\begin{array}{l}\text { Service d'Anesthésie Réanimation } \\
\text { Hôpital COCHIN } \\
27 \text { R FAUBOURG ST-JACQUES } \\
75014 \text { PARIS }\end{array}$ \\
\hline $\begin{array}{l}\text { PETITPAS, Franck } \\
\text { MIMOZ Olivier } \\
\text { NANADOUMGAR Hodanou }\end{array}$ & $\begin{array}{l}\text { Service de Réanimation Chirurgicale } \\
\text { HÔPITAL DE LA MILETRIE } \\
2 \text { RUE DE LA MILETRIE } \\
\text { B. P. } 577 \\
86021 \text { POITIERS }\end{array}$ \\
\hline $\begin{array}{l}\text { CHASTRE, Jean } \\
\text { COMBES Alain } \\
\text { NIESZKOWSKA Ania }\end{array}$ & $\begin{array}{l}\text { Service de Réanimation Médicale } \\
\text { C.H.U. PITIE SALPETRIERE } \\
\text { 47 BOULEVARD DE L'HOPITAL } \\
75013 \text { PARIS }\end{array}$ \\
\hline $\begin{array}{l}\text { AMATHIEU Roland } \\
\text { LEVESQUE Eric }\end{array}$ & $\begin{array}{l}\text { Réanimations et surveillance continue } \\
\text { chirurgicales HOPITAL HENRI MONDOR } \\
51 \text { AV DE LATTRE DE TASSIGNY } \\
94010 \text { CRETEIL }\end{array}$ \\
\hline COOK Fabrice & $\begin{array}{l}\text { Réanimations et surveillance continue } \\
\text { chirurgicales } \\
\text { HOPITAL HENRI MONDOR } \\
51 \text { AV DE LATTRE DE TASSIGNY } \\
94010 \text { CRETEIL }\end{array}$ \\
\hline $\begin{array}{l}\text { CONSTANTIN, Jean-Michel } \\
\text { CHARTIER Christian } \\
\text { JABAUDON Mathieu } \\
\text { PERBET Sébatien }\end{array}$ & $\begin{array}{l}\text { Service d'Anesthésie et réanimation } \\
\text { HÔPITAL HOTEL DIEU } \\
\text { Boulevard Léon Malfreyt } \\
\text { 63058 CLERMONT FERRAND CEDEX } 1\end{array}$ \\
\hline COLIN, Gwenhaël & $\begin{array}{l}\text { Service de Réanimation } \\
\text { CENTRE HOSPITALIER RENE DUBOS } \\
6 \text { avenue de I'lle de France } \\
95303 \text { CERGY PONTOISE CEDEX }\end{array}$ \\
\hline RIGAUD Jean-Philippe & $\begin{array}{l}\text { Service de Réanimation Polyvalente } \\
\text { CENTRE HOSPITALIER DIEPPE } \\
\text { AVENUE PASTEUR, BP } 219 \\
76200 \text { DIEPPE, FRANCE }\end{array}$ \\
\hline $\begin{array}{l}\text { HAOUACHE Hakim } \\
\text { KAMOUN Walid } \\
\text { SLAVOV Velislav }\end{array}$ & $\begin{array}{l}\text { Réanimations et surveillance continue } \\
\text { chirurgicales } \\
\text { HOPITAL HENRI MONDOR } \\
51 \text { AV DE LATTRE DE TASSIGNY } \\
94010 \text { CRETEIL }\end{array}$ \\
\hline $\begin{array}{l}\text { LORIFERNE, Jean-François } \\
\text { LE FLOCH Anne Sophie }\end{array}$ & $\begin{array}{l}\text { Service de Réanimation } \\
\text { HOPITAL SAINT-CAMILLE - BRY S/M } \\
2 \text { R DES PERES CAMILLIENS } \\
94366 \text { BRY SUR MARNE }\end{array}$ \\
\hline JOANNES BOYAU, Olivier & $\begin{array}{l}\text { Service d'Anesthésie Réanimation } \\
\text { HOPITAL DU HAUT LEVEQUE } \\
\text { Avenue de Magellan } \\
33604 \text { PESSAC }\end{array}$ \\
\hline BRIVET, François & $\begin{array}{l}\text { Service de Réanimation Médicale } \\
\text { Polyvalente } \\
\text { Hôpital ANTOINE BECLERE } \\
157 \text { R DE LA PTE DE TRIVAUX } \\
92140 \text { CLAMART }\end{array}$ \\
\hline
\end{tabular}




\section{Surname and name of sites principal investigator}

Ricome, Jean-Louis

Slama, Michel

Leroy, Olivier

Capellier, Gilles

Mantz, Jean

D’Honneur, Gilles

Brenas, François

Benhamou, Miche

Starczala, Eric

BOUGON David

\section{Study sites details}

Service de Réanimation médico-chirurgicale-SMUR

C.H.I POISSY-SAINT GERMAIN

10 Rue du CHAMP GAILLARD

BP 3082

78303 POISSY

Service de Réanimation Médicale Sud CENTRE HOSPITALIER LYON-SUD 69495 PIERRE BENITE CEDEX

Service de Néphrologie GROUPE HOSPITALIER SUD

AV RENE LAENNEC 80054 AMIENS

Service de Réanimation Médicale et

Maladies Infectieuses

C.H. TOURCOING GUSTAVE DRON

155 rue DU PRESIDENT COTY

BP619

59208 TOURCOING

Service de Réanimation Médicale - SAMU 25

HOPITAL JEAN MINJOZ

3 BD ALEXANDRE FLEMING

25030 BESANCON

Service de Anesthésie Réanimation

Chirurgicale

Hôpital ANTOINE BECLERE

157 R DE LA PTE DE TRIVAUX

92140 CLAMART

Service de Réanimation anesthésie

CHU DE REIMS

45 rue Cognacq-Gay

51092 REIMS CEDEX

Service d'Anesthésie Réanimation SMUR Hôpital BEAUJON

100 BD DU GENERAL LECLERC

92110 CLICHY

Service d'Anesthésie Réanimation

Hôpital JEAN VERDIER

AV DU 14 JUILLET

93140 BONDY

Service d'Anesthésie-Réanimation

CENTRE HOSPITALIER

BD DU DR.CHANTEMESSE

43012 LE PUY EN VELAY CEDEX

Service de Réanimation Cardiaque

HOPITAL PRIVE D'ANTONY

1 RUE VELPEAU

92166 ANTONY CEDEX

Service de Réanimation Médicale CENTRE HOSPITALIER LEON BINET DE PROVINS

Route de CHALAUTRE

774888 PROVINS CEDEX

Service de réanimation

1 AVENUE DE L'HOPITAL

74370 EPAGNY METZ-TESSY

\section{Author details}

${ }^{1}$ General ICU, Service de Réanimation, Hôpital Raymond Poincaré, Laboratory of Infection and Inflammation, U1173, AP-HP, University of Versailles SQY and INSERM, 104 Boulevard Raymond Poincaré, 92380 Garches, France. ${ }^{2}$ Service de Réanimation Médicale, Hôpital Henri Mondor, AP-HP, Créteil, France.

${ }^{3}$ Service de Réanimation Médicale, Hôpital Cochin, AP-HP, Paris, France.

${ }^{4}$ Service d'Anesthésie Réanimation, Hôpital Nord, AP-HM, Marseille, France.

${ }^{5}$ Service de Médecine Intensive et Réanimation, Hôpital Saint-Joseph, AP-HP, Université Paris Descartes, Paris Sorbonne Cité, Paris, France. ${ }^{6}$ Service de Pharmacologie, Centre d'Investigation Clinique INSERM 1414, CHU de Rennes, Université de Rennes 1, Rennes, France. ${ }^{7}$ Département des Essais Cliniques, AGEPS, Paris, France. ${ }^{8}$ Délégation à la Recherche Clinique, Hôpital Saint-Louis, AP-HP, Paris, France.

The online version of the original article can be found unde doi:10.1186/s13613-016-0147-3.

Received: 27 June 2016 Accepted: 1 July 2016

Published online: 19 August 2016

\section{Reference}

1. Annane D, Buisson CB, Cariou A, Martin C, Misset B, Renault A, Lehmann $B$, Millul V, Maxime V, Bellissant E, APROCCHSS Investigators for the TRIGGERSEP Network. Design and conduct of the activated protein $C$ and corticosteroids for human septic shock (APROCCHSS) trial. Ann Intensive Care. 2016:6:43. doi:10.1186/s13613-016-0147-3.

We are sorry for any inconvenience this has caused. 